\title{
Erratum
}

\author{
S. Beuchler ${ }^{\star} \cdot$ R. Schneider ${ }^{\star}$ C C. Schwab ${ }^{\star \star}$
}

\section{Multiresolution weighted norm equivalences and applications}

Published online: 15 November 2005

(C) Springer-Verlag 2005

Numer. Math (2004)

Digital Object Identifier (DOI) 10.1007/s00211-003-0491-8

Published online: April 8, 2004 - (c) Springer-Verlag 2004

Four equations in statements and proofs of Theorems 3.2 and 3.3 were printed with errors. The text below contains the correct equations.

Theorem 3.2 The infinite matrix $M=\left(\left(\psi_{k}^{l}, \psi_{k^{\prime}}^{l^{\prime}}\right)_{w}\right)_{(k, l) ;\left(k^{\prime}, l^{\prime}\right)}$ is bounded in $l_{2}$.

Proof We decompose the matrix $M$ into $M=M_{1}+M_{2}$ where the coefficients in $M_{2}$ are $\left(\psi_{k}^{l}, \psi_{k^{\prime}}^{l^{\prime}}\right)_{w}$ iff $0 \in \operatorname{supp} \psi_{k}^{l} \cap \operatorname{supp} \psi_{k^{\prime}}^{l^{\prime}}$ and $M_{1}$ does not contain the interaction of wavelets which are both located at the point zero. By applying Theorem 3.1, Lemma 3.7 and the Schur Lemma to $M_{1}$ we have $\left\|M_{1}\right\|_{2} \leq c$. From Lemma

* Supported by the DFG-Sonderforschungsbereich 393 "Numerische Simulation auf massiv parallelen Rechnern".

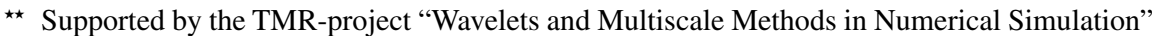
of the European Union and by the swiss Government under Grant No. BBW 97.404.

\footnotetext{
S. Beuchler

Johann Radon Institute for Computational and Applied Mathematics, Austrian Academy of Science, Altenberger Strasse 69, 4040 Linz, Austria

E-mail: sven.beuchler@oeaw.ac.at

R. Schneider

Scientific Computing, Institute of Computer Science, Christian Albrecht University Kiel, Christian-Albrechts-Platz 4, 24098 Kiel, Germany

E-mail: rs@numerik.uni-kiel.de

C. Schwab (凶)

Seminar für Angewandte Mathematik, Eidgenössische Technische Hochschule, 8092 Zürich, Switzerland

E-mail: schwab@math.ethz.ch
} 
3.8 we have $\left\|M_{2}\right\|_{1} \leq c$ and $\left\|M_{2}\right\|_{\infty} \leq c$ which shows $\left\|M_{2}\right\|_{2} \leq c$. Hence, the assertion is proven.

We show now the equivalence of the $L_{w}^{2}$ norm of a function

$$
u=\sum_{l=l_{0}}^{\infty} \sum_{k} u_{k}^{l} \psi_{k}^{l} \in L_{w}^{2}((0,1))
$$

with its discrete $l_{w}^{2}$ norm of the coefficients $\left(u_{k}^{l}\right)_{(k, l)} \in \mathbb{R}$, i.e.

$$
\left\|\left|u_{k}^{l}\right|\right\|_{w}^{2}:=\sum_{l} \sum_{k} w^{2}\left(2^{-l} k\right)\left|u_{k}^{l}\right|^{2} .
$$

Theorem 3.3 Let us assume that Assumptions 3.1 and 3.2 are valid. For any function $u=\sum_{l=l_{0}}^{\infty} \sum_{k} u_{k}^{l} \psi_{k}^{l} \in L_{w}^{2}((0,1))$ holds

$$
\|u\|_{w}^{2} \approx\left\|\mid u_{k}^{l}\right\| \|_{w}^{2}
$$

Proof From Theorem 3.2 we conclude

$$
\begin{aligned}
\|u\|_{w}^{2} & =\sum_{l, l^{\prime}} \sum_{k, k^{\prime}} u_{k}^{l} u_{k^{\prime}}^{l^{\prime}} w\left(2^{-l} k\right) w\left(2^{l^{\prime}} k^{\prime}\right)\left(\psi_{k}^{l}, \psi_{k^{\prime}}^{l^{\prime}}\right)_{w} \\
& \leq\|M\|_{2} \sum_{l} \sum_{k}\left(\left|u_{k}^{l}\right| w\left(2^{-l} k\right)\right)^{2} \leq c\left|\left\|u_{k}^{l} \mid\right\|_{w}^{2} .\right.
\end{aligned}
$$

To prove the lower estimate we consider the dual system

$$
\widetilde{v}=\sum_{l} \sum_{k} \widetilde{v}_{k}^{l} \widetilde{\psi}_{k}^{l}=G\left(\widetilde{v}_{k}^{l}\right)
$$

in the dual space $L_{w^{-1}}^{2}((0,1))$. We denote by $\tilde{M}$ the mass matrix of the dual wavelet basis $\widetilde{\psi}_{k}^{l}$ with respect to the $L_{w^{-1}}^{2}((0,1))$ innerproduct. Then, by the same arguments

$$
\|\widetilde{v}\|_{w^{-1}}^{2} \leq\left.\|\widetilde{M}\|_{2}\left\|\widetilde{v}_{k}^{l}\right\|\right|_{w^{-1}} ^{2}
$$

This means $G: l_{w^{-1}}^{2} \rightarrow L_{w^{-1}}^{2}((0,1))$ is bounded. Therefore, the adjoint operator $G^{*}: L_{w}^{2}((0,1)) \rightarrow l_{w}^{2}$ is bounded, too. $G^{*}$ is explicitly given by

$$
G^{*} u:=\left(\left\langle u, \widetilde{\psi}_{k}^{l}\right\rangle\right)_{l, k}=\left(u_{k}^{l}\right)_{l, k}
$$

which proves the lower bound.

The online version of the original article can be found at http://dx.doi.org/10.1007/s00211-003-491-8 